\title{
THE DIAGNOSIS OF CORRECTED TRANSPOSITION OF THE GREAT VESSELS
}

\author{
BY \\ MARÍA V. DE LA CRUZ,* BURTON J. POLANSKY, † AND \\ FRANCISCO NAVARRO-LOPEZ
}

From the National Institute of Cardiology, Mexico City, Mexico

Received Oct. 15, 1961

Accurate diagnosis of corrected transposition of the great vessels has become important since the progress in cardiac surgery. Corrected transposition without any other cardiac abnormality is of no clinical significance, but more often is accompanied by other cardiac abnormalities, most frequently pulmonary stenosis and interventricular septal defects (Anderson et al., 1957), when an accurate diagnosis becomes of great clinical importance since operation will often be considered. The failure to recognize this entity before operation may cause considerable difficulty at operation since a different approach may be required (Lillehei et al., 1953). The diagnosis may be overlooked by the cardiologist and even at operation, and the surgeon may lose valuable time identifying the anatomical situation before he can decide on what type of correction he is able to offer.

We therefore analyse an example of each of the two major possibilities in the simple classification that one of us has proposed for corrected transposition of the great vessels (De la Cruz et al., 1959) and will present the general rules that are of value in the clinical study and diagnosis of this malformation. Finally we present a method that can be used to localize the chambers of the heart not only in a corrected transposition but in any situation in which adequate observations can be made.

\section{EMBRYOLOGY}

Corrected transposition of the great vessels is a congenital malformation of the heart in which there is transposition of the great vessels corrected by a change in the relation between atria and ventricles, permitting the venous atrium to communicate with the pulmonary trunk across the anatomical left ventricle, and the arterial atrium to communicate with the aorta across the anatomical right ventricle.

Isolated transposition of the great vessels is characterized by the aorta arising anterior and parallel to the pulmonary trunk (De la Cruz et al., 1956). Consequently the aorta is seen arising anterior to the crista supraventricularis from the anatomical right ventricle and the pulmonary artery arises in a posterior position from the anatomical left ventricle. The great vessels are parallel in their course and do not cross as in the normal situation.

The detailed embryology of corrected transposition of the great vessels has been given in a recent work (De la Cruz et al., 1959), published by this laboratory, therefore in this paper we shall pnly present a brief review of the points most pertinent to an understanding of the problem.

\footnotetext{
* Director of the Department of Embryology. National Institute of Cardiology of Mexico.

$\dagger$ Post-doctoral Research Fellow of the U.S.P.H.S. National Heart Institute at the National Institute of Cardiology of Mexico.

$\ddagger$ Fellow, National Institute of Cardiology of Mexico.
} 


\section{Material AND Method}

The congenital heart specimens collected in the Instituto Nacional de Cardiología de México $\overrightarrow{\vec{D}}$ number 214, and 4 have corrected transposition of the great vessels. Since we were interested primarily in clarifying and simplifying the concept of corrected transposition we selected only two cases, each representative of one of the two major groups of corrected transposition that one of us $\overline{\vec{p}}$. has described (De la Cruz et al., 1959). Two methods of special study were employed with a careful $\widehat{\Omega}_{\Omega}^{\circ}$ and detailed anatomical review.

First, we placed catheters in the heart as they might be situated at the time of catheterization $\stackrel{5}{\circ}$ and we X-rayed the specimens in the frontal and left lateral position. Secondly we filled the hearts: with contrast medium and again X-rayed in the frontal and left lateral positions, so as to simulate $\vec{\omega}$ an in vivo angiocardiogram. The contrast medium was a heated liquid mixture of lipiodol and $\stackrel{\mathcal{F}}{5}$ paraffin which was poured into the various chambers of the open heart and quickly chilled in order to solidify and mould the paraffin to the form of the respective ventricle and great vessel. We thus? could correlate accurately the position of the contrast material with the anatomy of the specimen. + +

Finally we performed the same type of studies on two hearts that could be confused with our $\stackrel{\infty}{\infty}$

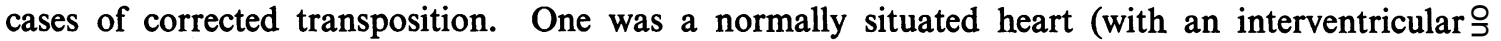
septal defect) and the other was a heart with mirror image dextrocardia (with tetralogy of Fallot). $\overrightarrow{ }$

The electrocardiograms from the two hearts with corrected transposition were reviewed and correlated with the position of the atria in each case during life.

\section{RESULTS}

\section{1.-Corrected Transposition of the Great Vessels in a Normally Situated Heart}

Anatomy. The atrium situated on the right side receives both inferior and superior vena cava. This atrium has a prominent group of pectinate musculature which originates in the atrial appendage and ends in the crista terminalis which is in a right paramedial position. The limbus of the foramen, ovale is on the right side of the inter-atrial septum in this chamber (Fig. 1A). This is therefore the anatomical right atrium and is situated on the right side and communicates via a bicuspid valve with the posterior ventricle which has two groups of papillary muscles, one anterior and one posterior. The superior two-thirds of the septal surface of this ventricle is smooth (Fig. 1A and 2C) and there is no crista supraventricularis, so that this ventricle has all the characteristics of the anatomical left ventricle though situated on the right side. The pulmonary trunk arises from this ventricle and is posterior, to the right, and parallel to the aorta throughout its entire course (Fig. 2A, B, C).

The atrium that is situated on the left receives the four pulmonary veins and has a smooth interior surface (Fig. 1B) and is therefore the anatomical left atrium: it communicates via a tricuspid valve with the ventricle that is anterior, has a crista supraventricularis, a moderator band, an anterior papillary muscle, and its septal surface is trabeculated (Fig. 1B, and 2D). This ventricle therefore has all the characteristics of the anatomical right ventricle even though it is situated on the left. The aorta arises from this ventricle and is anterior, to the left, and parallel to the pulmonary trunk throughout its entire course (Fig. 2A, B, D). In our specimen there is also a basal medial interventricular septal defect and an atrial septal defect of the foramen ovale type. The viscera are normal in position and the apex of the heart is to the left.

The final anatomical diagnosis is corrected transposition of the great vessels in a normally situated heart, with a basal medial ventricular septal defect and an atrial septal defect of the foramen ovale type.

Catheterization. In the frontal view the catheter on the right passes from the inferior vena cava through the right-sided atrium and ventricle to one of the branches of the pulmonary trunk (Fig. $3 \mathrm{~A}$ ). Note that the catheter leaves the ventricle in a straight and not an oblique manner, which is $\stackrel{\mathbb{D}}{2}$ quite different from the route of the catheter in the control heart without transposition (Fig. 3B). If the left side of the heart is catheterized at the same time one may see from the course of the catheter 


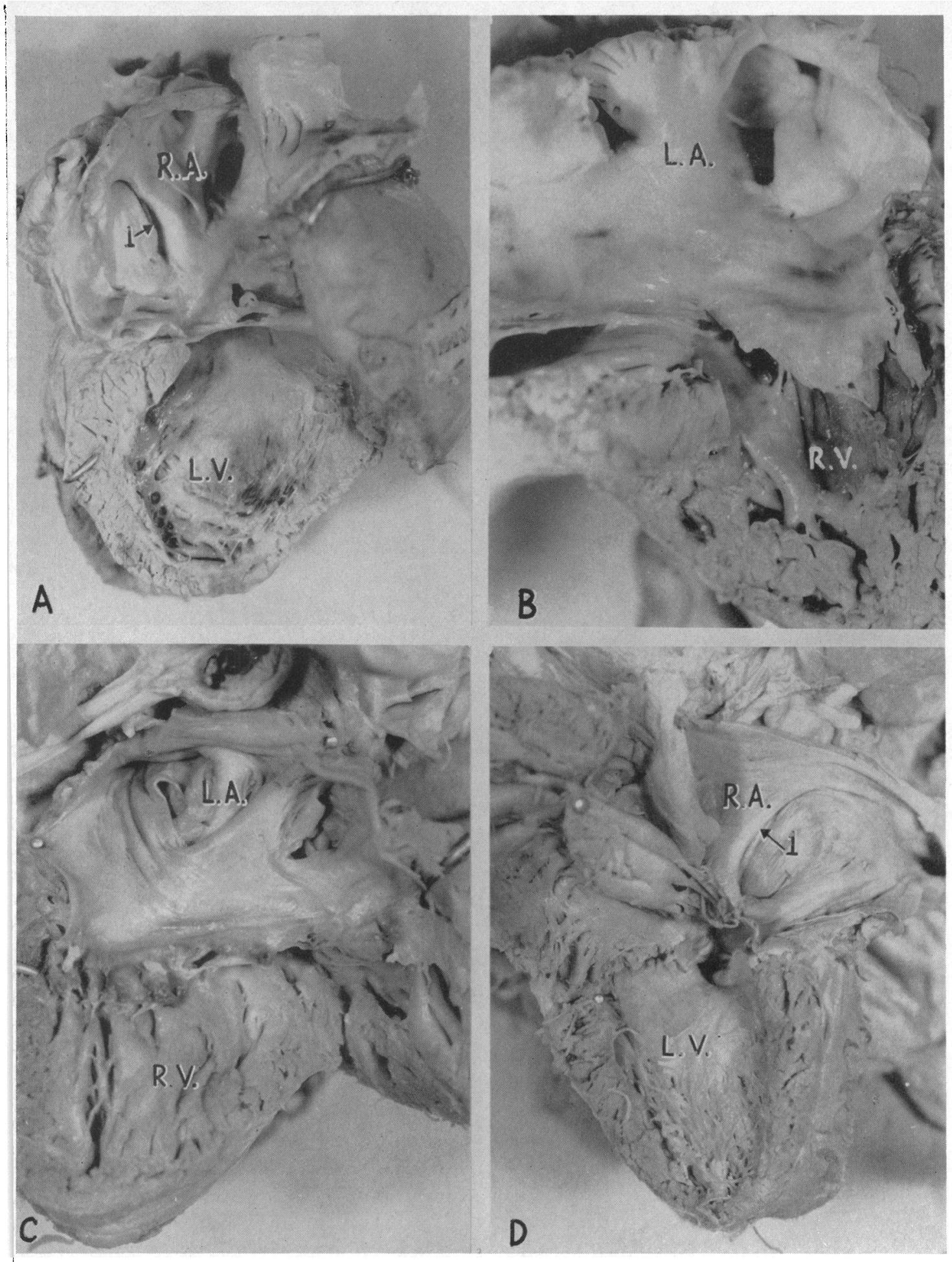

FIG. 1.-Atrio-ventricular relationships in corrected transposition of the great vessels with the atria and ventricles opened. (A) Corrected transposition of the great vessels in a normally situated heart viewed from the right side. Atrium on the right has the characteristics of the anatomical right atrium (R.A.) and joins the right-sided ventricle which has the characteristics of an anatomical left ventricle (L.V.). (B) Same specimen as in (A), viewed from left. The left-sided atrium has the characteristics of a left atrium (L.A.) and joins to the left-sided ventricle which has the anatomical characteristics of the right ventricle (R.V.). (C) Corrected transposition of the great vessels in a heart with mirror image dextrocardia viewed from the right side. The right-sided atrium shows the characteristics of the anatomical left atrium and joins to the right-sided ventricle which has the anatomical characteristics of the right ventricle. (D) Same specimen as in $\mathrm{C}$ viewed from the left side. The left-sided atrium has the characteristics of the right atrium and joins to the left-sided ventricle which has the anatomical characteristics of the left ventricle. 


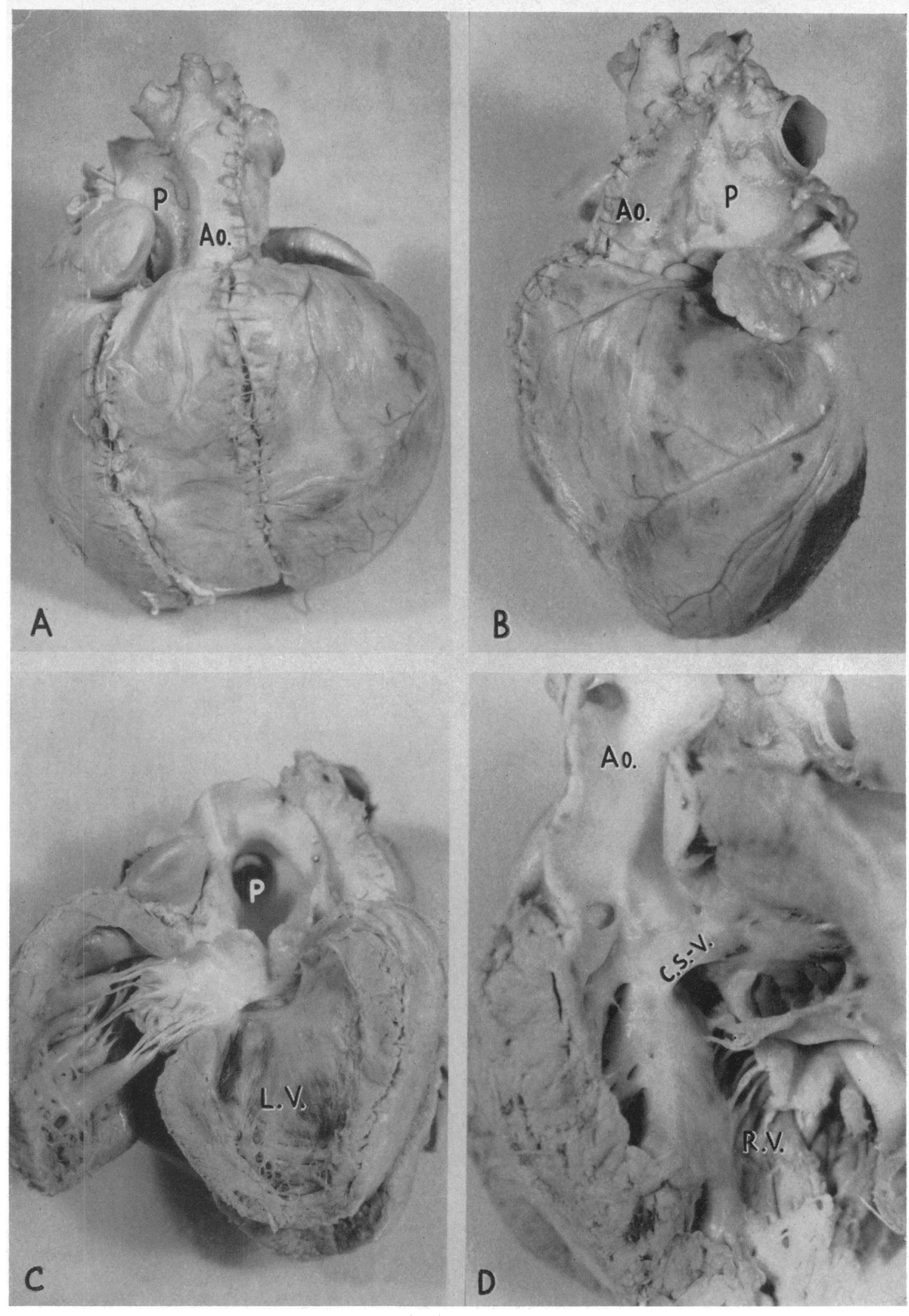

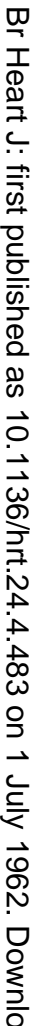



음

음

吾 

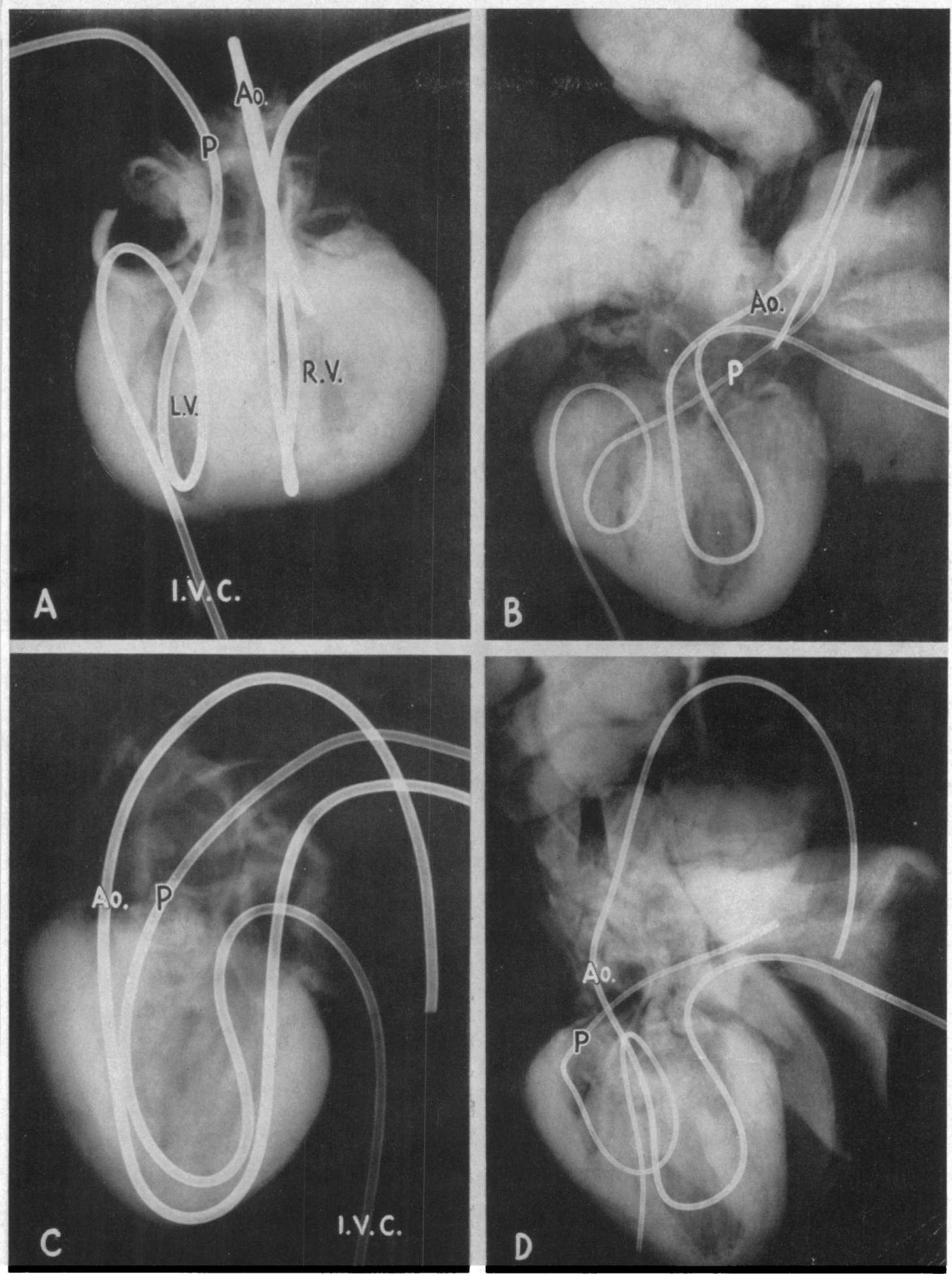

FIG. 3.-Post-mortem catheter studies in a normally situated heart with corrected transposition of the great vessels $(A, C)$, and in a normally situated heart $(B, D)$ as a control. (A) Corrected transposition, frontal view; both catheters parallel and straight. Aorta on the right and pulmonary trunk on the left. (B) Control heart, frontal view; catheter in the pulmonary trunk crosses the catheter in the aorta. Note that both catheters arise from their ventricles in an oblique manner. (C) Corrected transposition, lateral view. Aorta anterior and parallel to the pulmonary trunk which arises posteriorly. (D) Control heart, lateral view. Pulmonary trunk arises anterior and crosses the aorta which arises posteriorly. The lateral view (C) establishes the diagnosis of transposition of the great vessels as the aorta is anterior and parallel to the pulmonary trunk. The anterior aorta must arise from the anterior ventricle which is always anatomically a right ventricle. Since the aorta arises on the left (frontal view A) the left-sided ventricle is anatomically a right ventricle. I.V.C.=Inferior vena cava. 

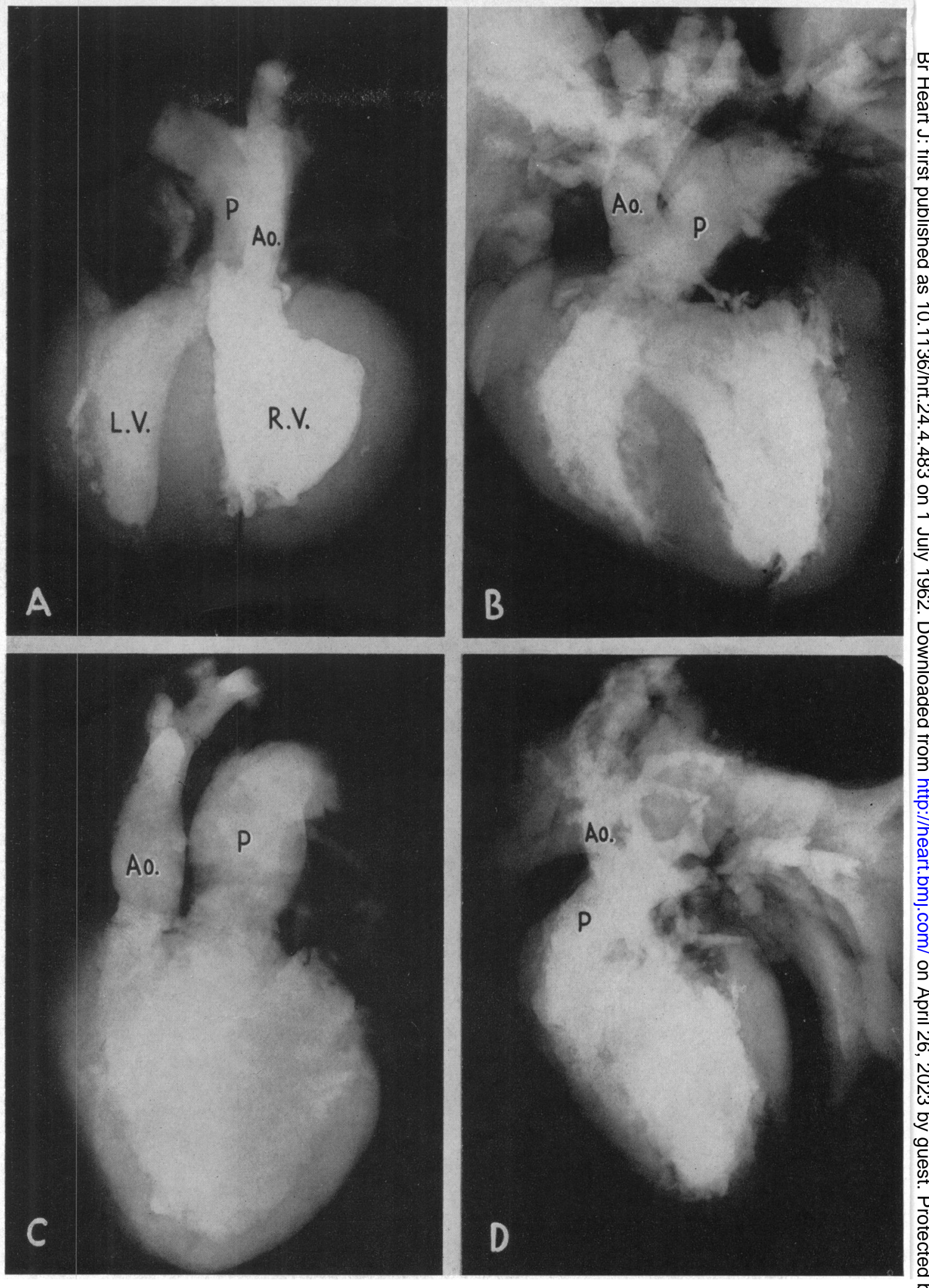

Fig. 4.-Post-mortem angiocardiographic studies of the same specimen as in Fig. 3. (A) Corrected transpositionfrontal view. Both great vessels arise in a straight manner and are parallel. Aorta on the left and pulmonary trunk on the right. (B) Control heart-frontal view. Pulmonary trunk crosses the aorta. Both vessels arise obliquely. (C) Corrected transposition-lateral view. Aorta anterior and parallel to the pulmonary trunk. (D) Control heart-lateral view. Pulmonary trunk arises anterior and crosses the aorta which arises posteriorly. As there is transposition of the great vessels (aorta anterior in C) the aorta must arise from the anterior ventricle which is always anatomically a right ventricle. Since the aorta in A (frontal view) is on the left the anatomical right ventricle is on the left side. 

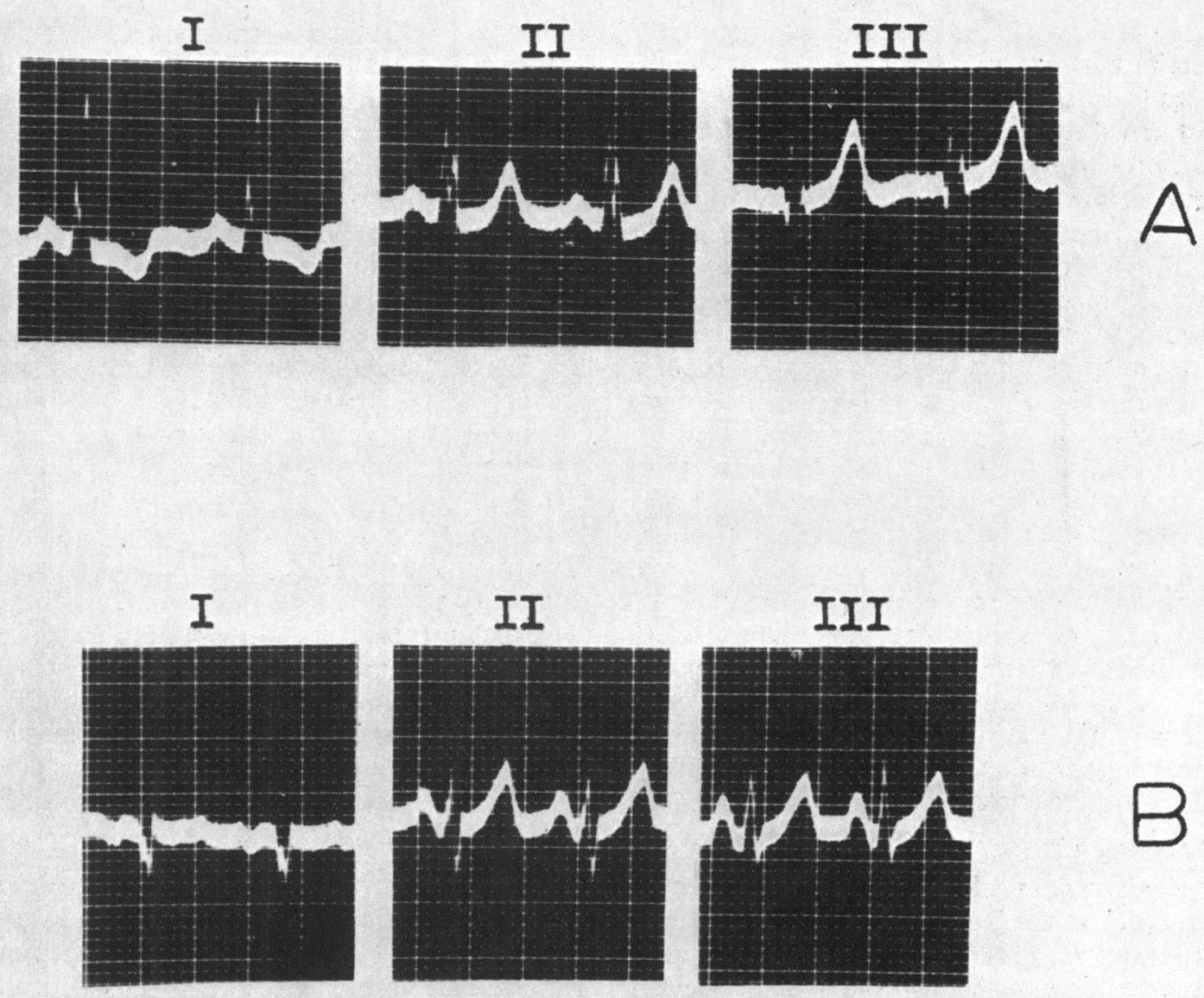

FIG. 5.-Electrocardiograms. Standard leads only. (A) Corrected transposition of the great vessels in a normally situated heart. Note positive $\mathbf{P}$ waves in leads I, II, and III. Axis of $\mathbf{P}+40^{\circ}$. (B) Corrected transposition of the great vessels in a heart with mirror image dextrocardia. Note negative $\mathbf{P}$ waves in lead $I$, positive $\mathbf{P}$ waves in leads II and III. Axis of $\mathbf{P}+110^{\circ}$.

that the aorta arises from the left-sided ventricle and is parallel to the pulmonary trunk (Fig. 3A). Finally in the heart without transposition both catheters arise in an oblique direction and their courses cross (Fig. 3B) in contrast with what is seen in hearts with transposition (Fig. 3A).

These relationships and positions are due to the fact that if the trunco-conal septum arises with its normal spiral rotation of $180^{\circ}$, the crossing and obliquity of the great vessels is obligatory. On the other hand, if the trunco-conal septum arises as a straight septum as in transposition, both vessels will not only be parallel but will also arise in a straight line (De la Cruz et al., 1956, 1959). Thus, the first strong suspicion of the presence of transposition during catheterization should come from the observation that the great vessel in which the catheter lies arises from its ventricle in a straight fashion, or that with simultaneous left and right catheterization the great vessels are parallel (Fig. 3A). Examination of the lateral films will establish the diagnosis with certainty. In the specimen with transposition (Fig. 3C) the aorta is seen arising anterior and parallel to the pulmonary trunk (compare with the lateral films of the heart without transposition shown in Fig. 3D), and the only possible diagnosis is then transposition of the great vessels.

Further analysis of the frontal plane X-rays bearing in mind the embryology (De la Cruz et al., $1956,1959)$ will allow one to deduce the position of the anatomical ventricles from their relation to 
the great vessels. The aorta always arises in an anterior position in transposition of the great vessels as shown in the lateral view (Fig. 2B and 3C). We know that the aorta always arises from the anterior ventricle, which is always the anatomical right ventricle, and since in our specimen the aorta arises from the ventricle that is on the left, we may conclude that the left-sided ventricle is the anatomical right ventricle (Fig. 2A, D, and 3A). In a similar manner, the pulmonary trunk in transposition of the great vessels always arises from the posterior ventricle which is always the anatomical left ventricle and since in our example the pulmonary trunk arises on the right, we may conclude that the right-sided ventricle is the anatomical left ventricle (Fig. 2A, C, and 3A).

The fundamental embryological observations that explain this situation are that the anatomical right ventricle may be either on the right or the left side depending on the type of bulboventricular loop, but it will always be anterior because it arises from the bulbus cordis which communicates with the aortic bulb, the anterior position of which is fixed throughout the developmental process of the heart because of its relationship to the aortic arches (De la Cruz et al., 1959). The anatomical left ventricle may also be on the right or left side of the adult heart, depending on the type of bulboventricular loop, but it will always be in a posterior position throughout because of its relationship to the atria which are fixed posteriorly (De la Cruz et al., 1959).

Finally, the relationship of the atria to the ventricles must be established to determine whether or not the transposition is corrected. Entry of the atrium on the right by a catheter from the inferior vena cava and passing the catheter to the pulmonary artery across the intervening ventricle establishes that the transposition is corrected (provided that a septal defect has not been traversed), because the anatomical right atrium (venous atrium) is shown to be in relation to the pulmonary trunk (Fig. 3A). We know that this is the anatomical right atrium because the inferior vena cava always enters the right atrium irrespective of its position. If the atrium is entered from the superior vena cava, whose relationship to the anatomical right atrium is much less certain, one cannot be sure of the position of the anatomical right atrium from the catheterization alone, but usually the electrocardiogram will be of great aid in determining the position of the right and left atria.

Angiocardiography. X-rays were taken of the same specimen filled with the solid contrast material. In the frontal view alone the diagnosis of the transposition is practically certain because the great vessels are parallel, do not cross, and arise in a straight fashion (Fig. 4A). The difference between this morphology and that of the heart without transposition is striking (Fig. 4B). As before in the lateral view the diagnosis is incontrovertible since the aorta is seen arising anterior and parallel to the pulmonary trunk (Fig. 4C). Compare with the normal heart (Fig. 4D). A second reference to the frontal views will enable one to localize the anatomical right ventricle in relation to the aorta on the left side and the anatomical left ventricle in relation to the pulmonary trunk on the right side (Fig. 4A). With this technique it is difficult to be certain of the position of the respective atria and greater reliance must be placed on the electrocardiogram.

Electrocardiography. The electrocardiographic picture of corrected transposition of the great $D$ vessels is not characteristic and is often confused by the association of other cardiac defects and so we will confine the discussion to its value, in sinus rhythm, of localizing the position of the anatomical $\mathbb{N}$ atria. The sinu-atrial node is always located in the anatomical right atrium and the activation of $N$ both atria proceeds from this focus, the anatomical right atrium being activated before the left atrium. Thus the activation wave in the atria passes from right to left in the heart which has the anatomical right atrium on the right and the anatomical left atrium on the left side. This means that in the normally situated atria the P wave will be positive in leads I, II, and III, whether the apex of the heart is on the left or the right (dextrorotation). If the atria are transposed as in mirrorimage dextrocardia, the anatomical right atrium with the sinu-atrial node will be on the left side and the anatomical left atrium will be on the right side. This activation wave will then spread from left to right and will produce a negative $P$ wave in lead I, and positive $P$ waves in leads II and III, whether the apex is on the left (some type of lævocardia if there is associated situs inversus) or on the right (mirror image dextrocardia). 

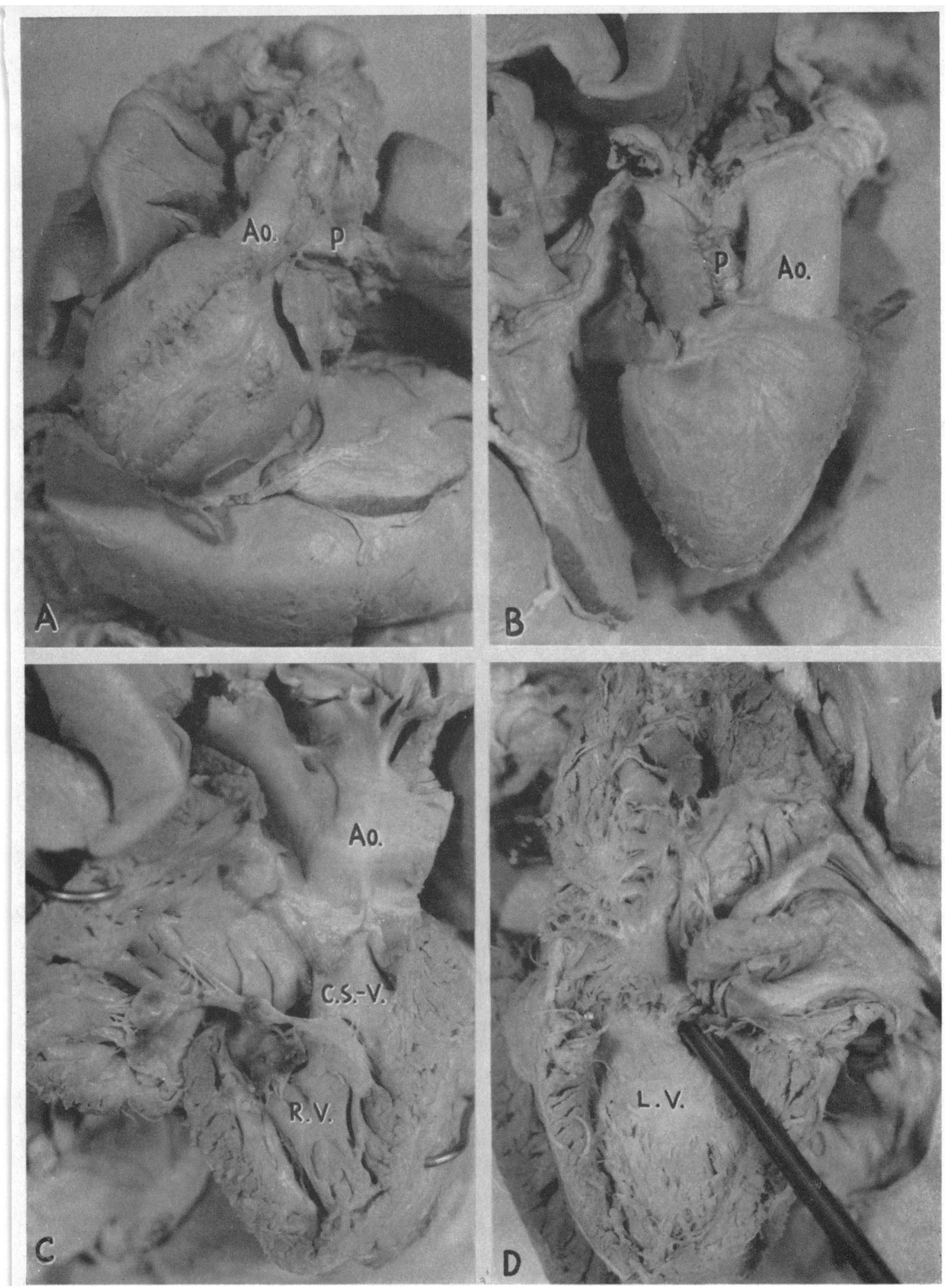

FIG. 6.-Corrected transposition of the great vessels in a heart with mirror image dextrocardia. (A) Frontal viewapex to the right, liver to the left (situs inversus). Aorta arises anterior, parallel and to the right of the pulmonary trunk. (B) Right lateral view-aorta anterior and parallel to the pulmonary trunk. (C) Right-sided ventricle opened showing the anatomical characteristics of right ventricle. Aorta arises from this ventricle anterior to the crista supraventricularis. (D) Left-sided ventricle opened showing the anatomical characteristics of the left ventricle. Probe placed in stenotic pulmonary artery showing that it arises from this ventricle. 


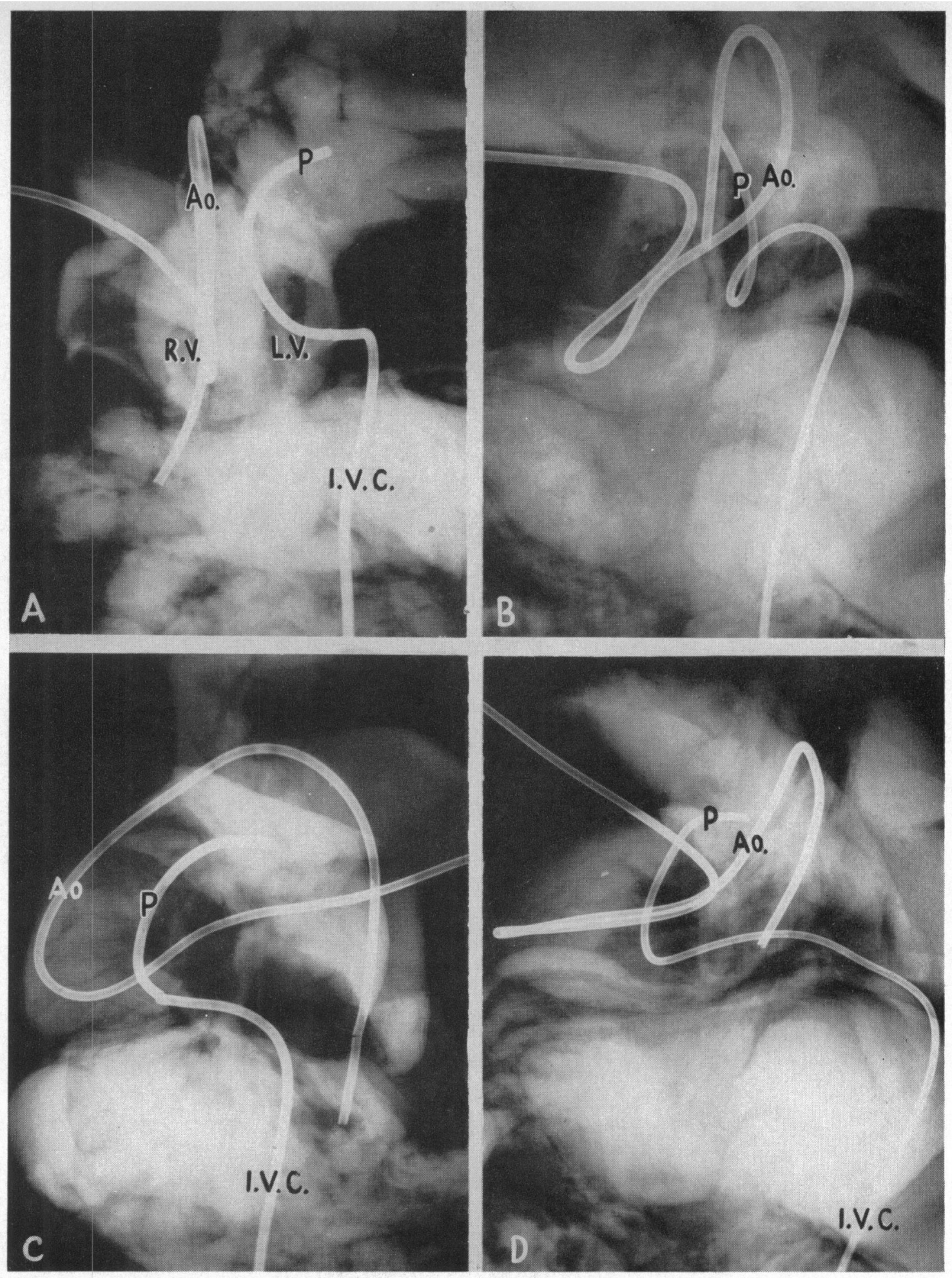

FIG. 7.-Post-mortem catheter studies in a heart with mirror image dextrocardia and corrected transposition of the great vessels $(A, C)$, with a control heart with mirror image dextrocardia and tetralogy of Fallot (B, D) (A) Corrected transposition - frontal view. Both catheters parallel and straight. Aorta on the right and pulmonary trunk on the left. (B) Control heart-frontal view. Catheter in pulmonary trunk crossing the catheter in the aorta. Note that both catheters arise from their ventricles in an oblique manner. (C) Corrected transpositionlateral view. Aorta anterior and parallel to the pulmonary trunk which arises posteriorly. (D) Control heartlateral view. Pulmonary trunk arises anterior and is about to cross the aorta which is posterior. The lateral view (C) establishes the diagnosis of transposition of the great vessels as the aorta is anterior and parallel to the pulmonary trunk. The anterior aorta must arise from the anterior ventricle which is always the anatomical right ventricle. Since the aorta arises on the right side (frontal view A) the right-sided ventricle is the anatomical right ventricle. 
Thus, the presence of positive $P$ waves in lead I means normally situated anatomical atria, and the presence of negative $P$ waves in lead I means that they are transposed. If there is nodal rhythm with normal position of both atria the P waves will be negative in leads II and III but positive in lead I; but if both atria are transposed and there is nodal rhythm the $\mathrm{P}$ wave will be negative in the three standard leads (Portillo et al., 1959). Therefore, the diagnostic value of the $\mathrm{P}$ wave in lead I remains the same either if there is sinus rhythm or nodal rhythm, i.e., a negative $\mathbf{P}$ wave in lead I suggests transposition of the atria. Fig. 5A shows the three standard leads of the specimen with corrected transposition of the great vessels in a normally situated heart: note that there is normal sinus rhythm and that the $\mathbf{P}$ waves are positive in leads I, II, and III, with the axis of the $\mathbf{P}$ wave $+40^{\circ}$. The interpretation of these findings was that the anatomical right atrium was on the right and the anatomical left atrium was on the left, thus completing the catheter and angiocardiographic data and leading to the diagnosis of corrected transposition of the great vessels in a normally situated heart.

\section{II.-Corrected Transposition of the Great Vessels with Mirror Image Dextrocardia}

Our example of corrected transposition of the great vessels in mirror image dextrocardia has an associated pulmonary stenosis. For this reason the heart chosen as a control was one with mirror image dextrocardia and an associated tetralogy of Fallot, a complex that is often incorrectly believed to be a type of transposition (Lev, 1953).

Anatomy. The atrium that is situated on the right side receives the four pulmonary veins and has a smooth interior surface. It is therefore the anatomical left atrium situated on the right side (Fig. 1C) and communicates by means of a tricuspid valve with the anterior ventricle that has a crista supraventricularis, a moderator band, and an anterior papillary muscle. The entire septal surface of this ventricle is trabeculated (Fig. 1C). This ventricle, therefore, has all the anatomical characteristics of the right ventricle and is situated on the right. The aorta arises from this ventricle anterior to the crista supraventricularis and is anterior, to the right, and parallel to the pulmonary trunk throughout its entire course (Fig. 6A, B, C). The aortic arch is on the right.

The atrium that is situated on the left receives both vena cava. It has a group of pectinate musculature which originates in the atrial appendage and ends in the crista terminalis which is in a left paramedial position. The limbus of the fossa ovalis is in this chamber (Fig. 1D). This is, therefore, the anatomical right atrium situated on the left. The anatomical right atrium communicates via a bicuspid valve with the posterior ventricle which has two groups of papillary muscles, one anterior and one posterior, and a septal surface that is smooth in its upper two-thirds (Fig. 1D). There is no crista supraventricularis, and it has all the characteristics of the anatomical left ventricle and is situated on the left. The pulmonary trunk, which is hypoplastic, arises from this ventricle and has a marked valvular stenosis with a bicuspid pulmonary valve (Fig. 6D). The pulmonary trunk is posterior, to the left, and parallel to the aorta throughout its entire course (Fig. $6 \mathrm{~A}, \mathrm{~B})$. There is a basal medial interventricular communication and a patent foramen ovale. The viscera are reversed (situs inversus) and the apex of the heart is to the right (Fig. 6A).

The final anatomical diagnosis is corrected transposition of the great vessels in a heart with mirror image dextrocardia, valvular stenosis, and hypoplasia of the pulmonary artery, basal medial interventricular communication, and probe-patent foramen ovale.

Catheterization. Fig. 7A, C, depicts the X-rays of the heart with corrected transposition of the great vessels and mirror image dextrocardia, with the catheters in place. In the frontal view the catheter on the left passes from the inferior vena cava through the left-sided atrium and ventricle to a branch of the pulmonary trunk (Fig. 7A). Note here also that the catheter leaves the ventricle in a straight and not oblique manner, which is different from the route of the catheter in the control heart with mirror image dextrocardia but without transposition (Fig. 7B). The catheter on the 

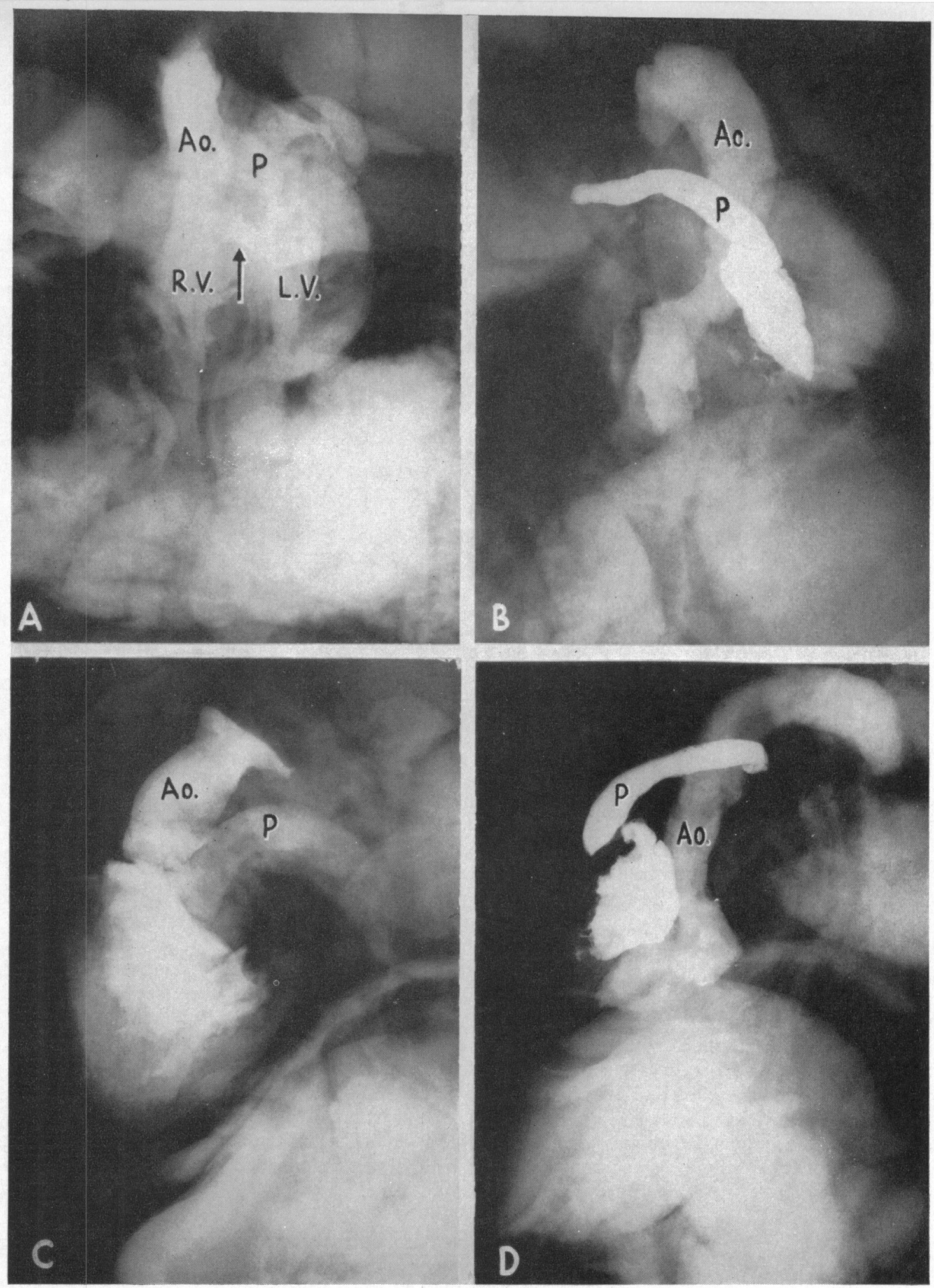

FIG. 8.-Post-mortem angiocardiographic studies of the same specimen as in Fig. 7. (A) Corrected transpositionfrontal view. Both great vessels arise in a straight manner and are parallel. Aorta on the right and pulmonary trunk on the left. (B) Control heart-frontal view. Pulmonary trunk crosses the aorta. Both vessels arise in oblique manner. Note pulmonary trunk has a greater concentration in this specimen. (C) Corrected transposition-lateral view. Aorta anterior and parallel to the pulmonary trunk which arises posteriorly. (D) Control heart -lateral view. Pulmonary trunk arises anterior and crosses the aorta which arises posteriorly. Pulmonary trunk has greater concentration in this specimen. As there is transposition of the great vessels (aorta anterior in C, lateral view), the aorta must arise from the anterior ventricle which is always anatomical right ventricle. Since the aorta in A (frontal view) is on the right, the anatomical right ventricle is on the same side. Arrow points to contrast material in the ventricular septal defect. 
right side of the heart enters the right-sided atrium and passes through the intervening right-sided ventricle to the aorta, but is parallel to the pulmonary trunk (Fig. 7A). Again in the heart without transposition both catheters cross and each one arises in an oblique fashion (Fig. 7B). If only one side is catheterized, the fact that the great vessel arises from its respective ventricle in a straight manner should arouse a strong suspicion of transposition. The left lateral X-rays show that the aorta is parallel and anterior to the pulmonary trunk (Fig. 7C), unlike the position of the vessels in the control heart (Fig. 7D). The diagnosis of transposition of the great vessels is now certain and we must refer to the frontal views to localize the position of the ventricles. From the embryological viewpoint, the ventricle that is on the left side, and from which the pulmonary trunk arises, must be the posterior ventricle and is therefore the anatomical left ventricle (Fig. 6D and 7A). The ventricle on the right side from which the aorta arises must be the anterior ventricle and is therefore the anatomical right ventricle (Fig. 6A, C, and 7A). Thus we have the anatomical right ventricle on the right and the left ventricle on the left in a heart which has its apex to the right and transposition of the great vessels. The definitive diagnosis rests on the position of the atria. Since the left-sided atrium was entered via the inferior vena cava this must be the anatomical right atrium (Fig. 7A), and the atrium on the right side must be the anatomical left atrium. One may also see from these films (Fig. 7A), that there is situs inversus, as the liver is on the left and the heart apex is on the right. It can be concluded that this is corrected transposition of the great vessels in a heart with mirror image dextrocardia.

Angiocardiography. Fig. 8A, C, shows the X-rays of the same specimen with transposition of the great vessels in a heart with mirror image dextrocardia filled with solid contrast material. The lesser concentration fills the left-sided ventricle and the pulmonary trunk, while the greater concentration fills the right-sided ventricle and the aorta (Fig. 8A). In the frontal view the diagnosis of transposition of the great vessels is practically certain because the great vessels are parallel, arising in a straight fashion from their respective ventricles (Fig. 8A). The difference between this morphology and that of the control heart is obvious (Fig. 8B). The definitive diagnosis is established from the lateral views where the aorta is seen arising anterior and parallel to the pulmonary trunk (Fig. 8C), unlike that of the control heart (Fig. 8D).

Further analysis of the frontal views, knowing the diagnosis of transposition, permits the correct identification of the anatomical right and left ventricle. Thus the aorta arises on the right and anterior, indicating that the right-sided ventricle must be the anterior ventricle which is always the anatomical right ventricle (Fig. 8A). The pulmonary trunk arises on the left and posterior, indicating that the left-sided ventricle must be the posterior one which is always the anatomical left ventricle (Fig. 8A). Thus we know from the angiocardiographic studies that there is a transposition of the great vessels in a heart in which the left-sided ventricle is the anatomical left ventricle giving origin to the pulmonary trunk and the right-sided ventricle is the anatomical right ventricle giving origin to the aorta (Fig. 6C, D). We also know that the apex of the heart is to the right and that there is situs inversus as well (Fig. 8A).

The definitive diagnosis depends on the relation between the atria and the ventricles. Since the position of the atria is difficult to determine from angiocardiography alone we must then turn to the electrocardiogram for information on this point.

Electrocardiography. The electrocardiogram of the specimen with corrected transposition of the great vessels in a heart with mirror image dextrocardia (Fig. 5B) shows sinus rhythm with $P$ waves which are negative in lead I, positive in leads II and III, and the axis of the P wave is $+110^{\circ}$. This means that activation in the atria is proceeding from left to right and that the anatomical right atrium is on the left side and the anatomical left atrium is on the right side. There is therefore mirror image positioning of the atria. Thus the electrocardiogram, when related to previous catheter and angiocardiographic findings, gives the diagnosis of corrected transposition of the great vessels in a heart with mirror image dextrocardia. 


\section{Discussion}

The respective anatomical atria can be found on either the right or left side, depending on the position in which they arise in the earliest stages of the heart as a straight tube. The atria are fixed posteriorly from the very beginning and cannot change their position (De la Cruz et al., 1959). One must think in terms, therefore, of the position of the anatomical atria and not in terms of either leftsided or right-sided atrium. The position of the anatomical atria can be derived from the catheter studies or from the $\mathbf{P}$ waves of the electrocardiogram.

In transposition of the great vessels the aorta is always parallel and anterior to the pulmonary trunk. For this reason the diagnosis of transposition is easily made from the lateral films with the catheter or the contrast material in place. The diagnosis may also be suspected from the frontal view, if the great vessels are parallel, and each, or one, arises from its respective ventricle in a straight line without crossing the other. It should be clear that in the uncomplicated form of transposition the aorta can only arise from the anterior ventricle (anatomical right ventricle), and the pulmonary trunk can only arise from the posterior ventricle (anatomical left ventricle).

The position of the respective ventricles in corrected transposition requires consideration. As with the atria the right and left anatomical ventricle may be either on the right or left side. One should think in terms of the position of the anatomical ventricle and not of right- and leftsided ventricle, because the ventricle arise from definitive embryological structures. The bulbus cordis, which always gives rise to the anatomical right ventricle, may be on the right or left side, but is always anterior since it is joined to other structures that are fixed anteriorly (aortic bulb). The anatomical right ventricle, therefore, is always anterior, though it may be either on the right or left side. The anatomical left ventricle (derived from the primitive ventricle which is always joined to the atria which are fixed posteriorly) may be on the right or left side, but must always be posterior (De la Cruz et al., 1959).

When we examine the frontal views of a case that we know has transposition of the great vessels (because the aorta is anterior and parallel to the pulmonary trunk in the lateral view) we can then identify the position of the anatomical right ventricle, because of its constant relation to the aorta. Therefore the anatomic right ventricle is on the right or the left, depending on whether the aorta is on the right or the left. Since the pulmonary trunk is always related to the posterior ventricle which is always the anatomical left ventricle, then this left ventricle is either on the right or left side depending on the position of the pulmonary trunk.

Finally the diagnosis of a transposition of the great vessels that is corrected rests on the relation of the atria to the ventricles. Thus an incorrect relation of the anatomical atria to the anatomical ventricles corrects the most important abnormal relation between the ventricles and the great vessels. The correction is brought about because the anatomical right atrium (venous atrium) communicates with the pulmonary trunk and the anatomical left atrium (arterial atrium) communicates with the aorta.

There are only two possible basic situations in which the relationships that we have outlined could occur (De la Cruz et al., 1959). Each of these has only two divisions, related to the position of the apex. The first group is that of corrected transposition of the great vessels in a normally situated heart or with its other division, dextrorotation (apex to the right): the hallmark in this group is that anatomically the right atrium is on the right and the left atrium is on the left. The other group is that of corrected transposition of the great vessels in mirror image dextrocardia, or its other division in some type of lævocardia (apex to the left): the hallmark of this group is that the anatomical right atrium is on the left and the anatomical left atrium is on the right as well as the presence of situs inversus.

Our method of analysis can be applied to the localization of all of the cavities of the heart in any given situation, i.e., transposition without correction, dextrocardia, dextrorotation, etc. The practical value is that in a complicated case the clinician may greatly aid the surgeon by clearly defining the anatomical situation pre-operatively thereby saving valuable time at operation and even permitting the avoidance of serious errors. Finally, there are other variations in the manner in 
which our observations may be used to study patients. For example, if only one side is catheterized and selective angiocardiography is employed from one of the great vessels, both vessels may be localized at the same time, avoiding the need for double catheterization.

\section{SUMMARY}

In a post-mortem study of anatomical, angiocardiographic, and catheterization data, we have compared two cases representative of the two principal groups of corrected transposition of the great vessels with two control cases that might be confused with them, and have applied this information, together with some obtained from the electrocardiogram, to the diagnosis in vivo of these congenital malformations.

The following observations were made.

(a) The $\mathrm{P}$ waves of lead I are of aid in determining the position of the anatomical atria.

(b) The diagnosis of transposition may be suspected from the frontal X-rays taken during catheterization or angiocardiography if one of the great vessels is seen arising in a straight line from its ventricle, or if both of the great vessels are seen to be parallel and uncrossed.

(c) The diagnosis of transposition can be made with certainty from the lateral films if the aorta is seen arising anterior and parallel to the pulmonary trunk.

(d) If the diagnosis of transposition is certain from the lateral views, reference to the frontal - views will localize the anatomical ventricles. Since the aorta is anterior it must arise from the anterior ventricle and this is always the anatomical right ventricle which will be on the right or left according to the position of the aorta. The pulmonary trunk is always posterior and arises from the posterior ventricle which is always the anatomical left ventricle: therefore the anatomical left ventricle will be localized on the right or left side according to the position of the pulmonary trunk. The frontal views will also show the position of the apex and the situation of the viscera.

By combining the information obtained with these studies, it is possible to localize accurately all the chambers of the heart and make the final diagnosis of corrected transposition of the great vessels in each of its varieties.

\section{REFERENCES}

Anderson, R. C., Lillehei, C. W., and Lester, R. G. (1957): Pediatrics, 20, 626.

De la Cruz, M. V., and Da Rocha, J. P. (1956). Amer. Heart J., 51, 782.

Anselmi, G., Cisneros, F., Reinhold, M., and Portillo, B. (1959). Amer. Heart J., 57, 104.

Lev, M. (1953). Autopsy Diagnosis of Congenitally Malformed Hearts. Charles C. Thomas, Springfield, Ill.

Lillehei, C. W., and Varco, R. L. (1953). Surgery, 34, 376.

Portillo, B., Anselmi, G., Sodi-Pallares, D., and Medrano, G. A. (1959). Amer. Heart J., 57, 396. 